\title{
Design and analysis of computer experiments
}

\author{
Sonja Kuhnt • David M. Steinberg
}

(C) Springer-Verlag 2010

\begin{abstract}
The design and analysis of computer experiments as a relatively young research field is not only of high importance for many industrial areas but also presents new challenges and open questions for statisticians. This editorial introduces a special issue devoted to the topic. The included papers present an interesting mixture of recent developments in the field as they cover fundamental research on the design of experiments, models and analysis methods as well as more applied research connected to real-life applications.
\end{abstract}

Keywords Computer experiments - Design of experiments - Latin hypercube designs $\cdot$ Gaussian process model

\section{Introduction}

The design and analysis of computer experiments has in recent years emerged as a new and important field in engineering, science and statistics. Due to increasing computer power and readily available codes, many physical processes can nowadays be simulated. The simulation code then serves as an efficient mode for exploring the properties of the process. In engineering, computer experiments replace or supplement expensive and time-consuming physical experiments. In some scientific areas, like nuclear engineering and climate research, computer simulators are the primary means for predicting the future effects of current policies. The design and analysis of computer experiments present a number of challenges for statistical research.

S. Kuhnt (殴

Faculty of Statistics, TU Dortmund University, 44221 Dortmund, Germany

e-mail: kuhnt@statistik.tu-dortmund.de

D.M. Steinberg

Department of Statistics and Operations Research, Tel Aviv University, Tel Aviv 69978, Israel

e-mail: dms@post.tau.ac.il 
Although the underlying computer code is often deterministic in nature, statistical methodology is essential. A careful selection of settings of input variables is needed for a systematic study of the change in the observed response. Computer codes are often complex, mirroring the real physical system that is being described. The analysis and management of the system therefore can often be greatly aided by building an approximate empirical model, known as a metamodel, which is much simpler than the true one. Moreover, random errors to the input of the computer model may need to be included to match randomness that will affect the real system. There has been a spurt of statistical research in this field in the last 20 years. Two books (Santner et al. 2003 and Fang et al. 2006) have been published summarizing the main recommendations for experimental design and modeling for computer experiments.

In this special issue we have collected seven papers. The first paper by Levy and Steinberg (2010) gives a general review of computer experiments. After identifying a wide range of applications, they describe the popular Gaussian process model, as well as possible alternatives and extensions. Calibration, tuning, validation and verification issues especially important for computer experiments are addressed. As the analysis of computer experiments naturally depends very much on the experimental design, the frequently used Latin hypercube designs and the idea of space-filling designs are introduced. Three papers in this issue specifically focus on various issues concerning Latin hypercube designs. A new algorithm to construct Latin hypercube designs in cases of inequality constraints on the input variables is developed by Petelet et al. (2010). Usually the choice of a design from the class of Latin hypercubes is based on applying specific design criteria. Here Jourdan and Franco (2010) introduce a new criterion based on the Kullback-Leibler information. This criterion measures how close the design points are to being uniformly distributed, aiming at a space-filling design. Pistone and Vicario (2010) compare different Latin hypercube designs by deriving the mean squared prediction error of the linear predictor in closed form. This involves the computation of the predictor variance in closed form which is solved via an algebraic statistical model. The contribution of Ratto and Pagano (2010) considers different modeling approaches within the framework of smoothing spline ANOVA. ACOSSO and SDR are presented individually and in a newly derived combination and are compared to the modeling approach using Gaussian process (Kriging) models. The final two papers of this issue are devoted to solving real-life applications. Wagner et al. (2010) develop a new procedure to model an induction heating process based on results simulated from a finite element model validated with experimental measurements. Lefebvre et al. (2010) deal with the problem of modeling aircraft InfraRed Signature, where the uncertainty of input parameters like meteorological conditions can not directly be incorporated into the computer simulation. They propose a three-step approach involving sensitivity analysis and Quasi-Monte Carlo estimation which finally leads to an appropriate neural network metamodel of the InfraRed Signature simulation code.

We are confident that the readers will enjoy this special issue and become interested in the application of and research on computer experiment models. The stimulus for this special issue was the ENBIS-EMSE 2009 Conference devoted to Computer Experiments at the Ecole Nationale Superieure des Mines de St-Etienne (France). We would like to thank O. Roustant and D. Ginsbourger for their great organization 
of that meeting as well as the Editors of the journal AStA Advances in Statistical Analysis, G. Kauermann and S. Lang, for the opportunity to organize this special issue.

\section{References}

Fang, K.T., Li, R., Sudjianto, A.: Design and Modeling for Computer Experiments. Chapman \& Hall/CRC, Boca Raton (2006)

Jourdan, A., Franco, J.: Optimal Latin hypercube designs for the Kullback-Leibler criterion. Adv. Stat. Anal. 94(4), 341-351 (2010)

Levy, S., Steinberg, D.M.: Computer experiments: A review. Adv. Stat. Anal. 94(4), 311-324 (2010)

Lefebvre, S., Roblin, A., Varet, S., Durand, G.: Metamodeling of aircraft infrared signature dispersion. Adv. Stat. Anal. 94(4), 405-422 (2010)

Petelet, M., Iooss, B., Asserin, O., Loredo, A.: Latin hypercupe sampling with inequality constraints. Adv. Stat. Anal. 94(4), 325-339 (2010)

Pistone, G., Vicario, G.: Comparing and generating Latin hypercube designs in Kriging models. Adv. Stat. Anal. 94(4), 353-366 (2010)

Ratto, M., Pagano, A.: Using recursive algorithms for the efficient identification of smoothing spline ANOVA models. Adv. Stat. Anal. 94(4), 367-388 (2010)

Santner, T.J., Williams, B.J., Notz, W.I.: The Design and Analysis of Computer Experiments. Springer, New York (2003)

Wagner, T., Bröcker, C., Saba, N., Biermann, D., Matzenmiller, A., Steinhoff, K.: Analysis of a thermomechanically coupled forming process using enhanced design and analysis of computer experiments. Adv. Stat. Anal. 94(4), 389-404 (2010) 